\title{
Effectiveness of PRP Injection in Reducing Recovery Time of Acute Hamstring Injury: A Critically Appraised Topic
}

\author{
Mary Lynn Manduca and Stephen J. Straub
}

\begin{abstract}
Clinical Scenario: Hamstring strains are common athletic injuries, with a high-recurrence rate (34\%). Recently, platelet-rich plasma (PRP) injections have gained popularity as a potential treatment option to accelerate healing of hamstring injury. Focused Clinical Question: Does the combination of PRP injection and rehabilitation decrease recovery time of acute hamstring injury as compared to rehabilitation alone in college athletes? Summary of Key Findings: A literature search resulted in 3 randomized controlled trials. One study showed benefits in various outcome measures with PRP, compared to rehabilitation alone, while 2 showed no benefits. One study reported improved pain, ultrasonography regenerative indications, and recovery time with PRP injection following acute hamstring injury; however, larger studies have shown no benefits. The literature demonstrates conflicting evidence regarding benefits of PRP injections in hamstring injuries. Clinical Bottom Line: At this time, PRP injections cannot be recommended as having value for hamstring injuries, compared to rehabilitation alone. Strength of Recommendation: Due to inconsistent or limited quality patient-oriented evidence in existing literature, the strength of this recommendation is grade B, based on the strength of recommendation taxonomy.
\end{abstract}

Keywords: rehabilitation, return to play, sport medicine, platelet-rich plasma

\section{Clinical Scenario}

Muscle injuries account for $30 \%$ to $50 \%$ of all athletic injuries, ${ }^{1}$ with hamstring strains accounting for $15 \% .^{2}$ Hamstring injuries are particularly common in endurance sports, soccer, rugby, hockey, football, and track - which has been attributed to the explosive and decelerating demands associated with such sports. ${ }^{3,4}$ Despite its prevalence, a universal treatment plan has yet to be established, but typically involves conservative treatment addressing strength, flexibility, and neuromuscular deficits as well as potential risk factors for hamstring injury through price, therapeutic exercise, electrotherapy, massage, mobility, and pharmacological interventions (ie, non-steroidal anti-inflammatory drugs). ${ }^{5}$ Despite these recommended treatment options, hamstring strains have the highest recurrence rate of all injuries. Estimates suggest that approximately one-third of hamstring strains experienced by college athletes were recurrent. ${ }^{6}$ A 2015 epidemiologic study investigating hamstring injury in 25 National Collegiate Athletic Association (NCAA) sports reported higher hamstring injury rates in men as compared to women for soccer, baseball/softball, and indoor track. Prior retrospective studies support this claim and suggest that decreased flexibility in males as a potential link to higher hamstring injury rates. Recently, platelet-rich plasma (PRP) injections have gained popularity as a possible treatment option to accelerate healing of muscle injury, specifically a hamstring injury. PRP injections are derived from an individual's centrifuged blood, which creates a serum that is highly concentrated with platelets, which contain several growth factors to assist in repair and regeneration. ${ }^{7}$ It has

Manduca and Straub are with the Department of Athletic Training/Sports Medicine, Quinnipiac University, Hamden, CT. Manduca (memanduca@quinnipiac.edu) is corresponding author. been suggested that PRP injections would create a higher than normal concentration of platelets in an injured area to promote healing and decrease recovery time. ${ }^{3}$ Despite the proposed benefits, the effect of PRP on hamstring injuries is unclear.

\section{Focused Clinical Question}

Does the combination of PRP injection and rehabilitation decreased recovery time of acute hamstring injury as compared to rehabilitation alone in college athletes?

\section{Summary of Search, Best Evidence Appraised, and Key Findings}

- The literature search was conducted to limit studies to those with level II evidence or higher that investigated the effect of PRP injection in addition to rehabilitation in the treatment of hamstring injury compared to rehabilitation alone.

- Three randomized controlled trials were included.

- All studies involved a comparison of PRP injections and rehabilitation to rehabilitation with no active injection. One study used a placebo saline injection in the comparison group. ${ }^{8,9}$

- All 3 studies included participants with acute hamstring injuries. ${ }^{1,9-11}$ Two studies ${ }^{9-11}$ included both grade I and II hamstring injury, while $1^{1}$ included only grade 2 a hamstring injury.

- Only 1 study reported that PRP injections following acute hamstring injury can decrease pain, increase ultrasonography regenerative indications, and decrease recovery time. ${ }^{1}$ Two studies showed no difference between groups. ${ }^{9-11}$ 


\section{Clinical Bottom Line}

While no adverse effects have been reported, the current literature demonstrates conflicting evidence regarding the benefits of including PRP injections in the treatment of acute hamstring injury. Therefore, at this time, PRP injections cannot be recommended as having value for hamstring injuries as compared to rehabilitative therapy alone. However, if a clinician chooses to include PRP injection in the treatment of a hamstring injury, there are several considerations that should be made. The results of this critically appraised topic (CAT) suggest that PRP injections may be more beneficial in younger-potentially high school and college agedhigher-level athletes who have sustained more severe acute hamstring injury, such as a grade IIa strain, or recurrent hamstring injuries.

It is also important to note that PRP injections are currently not prohibited in sport participation according to the World AntiDoping Agency. ${ }^{12}$ Intramuscular PRP administration was banned by the World Anti-Doping Agency until 2010, but the ban was lifted in 2011. ${ }^{13}$ According to the World Anti-Doping Agency, platelet-derived preparations like PRP were removed from the list of prohibited substances because current evidence does not demonstrate any potential improvements in sport performance beyond a potential therapeutic effect, despite the presence of some growth factors. However, individual growth factors remain on the prohibited list when given separately as "purified substances."

Another consideration is that due to the limitations and conflicts in the existing research, insurance companies currently do not cover PRP injections for patients. This means that a clinician will not receive third party reimbursement for administration of a PRP injection, and a patient will be required to directly cover the cost of injection. ${ }^{14}$ New reimbursement codes do not have an "assigned Medicare value," so the price of an injection is determined by individual clinicians based on their estimation of the work involved to provide the service, which can vary greatly, ranging from as low as $\$ 200$ to as high as $\$ 1500^{15}$ for a single injection.

\section{Strength of Recommendation}

Due to inconsistent or limited-quality patient-oriented evidence in existing literature, the strength of this recommendation is grade B, based on the strength of recommendation taxonomy. ${ }^{16}$

\section{Search Strategy}

\section{Terms Used to Guide Search Strategy}

- Patient/Client group: college-aged athletes with recurrent hamstring injury

- Intervention/Assessment: PRP injection

- Comparison: conservative treatment or rehabilitation program alone

- Outcomes: return to play (recovery time duration)

\section{Sources of Evidence Searched}

PubMed, Cochrane Library, CINAHL, SPORTDiscus, ScienceDirect, and SagePub were all searched using keywords PRP injection, hamstring, and hamstring injury. Additional resources were obtained by reference list reviews and individual searches.

\section{Inclusion and Exclusion Criteria}

\section{Inclusion Criteria}

- Investigated effect of PRP injection in addition to rehabilitation compared to rehabilitation alone for acute hamstring injury

- Recovery time (time to return to play) outcome measure

- Level II evidence or higher (based on Levels of Evidence, Oxford Centre for Evidence-Based Medicine ${ }^{17}$ ).

- Published after 2008

- Limited to human studies

- Limited to English language

- Limited to full text available

\section{Exclusion Criteria}

- Muscle injury not specific to hamstring

- Grade III hamstring injury

- Surgical treatment of hamstring injury

- Chronic hamstring injury ( $>7 \mathrm{~d}$ after onset)

\section{Results of Search}

A total of 3 applicable randomized controlled trials ${ }^{1,9-11}$ were selected for inclusion in this CAT. The selected studies are presented in Table 1.

\section{Best Evidence}

The studies presented in Table 2 were identified as the best evidence according to the inclusion and exclusion criteria. These studies best compared PRP and rehabilitation with rehabilitation alone as treatments for acute hamstring injury.

\section{Implications for Practice, Education, and Future Research}

The 3 studies included in this CAT presented conflicting evidence regarding the effect of PRP injection in combination with rehabilitation on recovery time in hamstring injury. Two articles showed no benefit of PRP injection in combination with rehabilitation compared to rehabilitation alone (total $\mathrm{N}=170$ ). ${ }^{9-11}$ One study

Table 1 Summary of Study Designs of Included Articles

\begin{tabular}{llcc}
\hline Level of evidence & Study design or methodology & Number included & References \\
\hline II & Single-blind randomized controlled trial & 1 & Hamid et al $^{1}$ \\
& Double-blind randomized placebo-controlled trial & 1 & Reurink et al $^{10,11}$ \\
& Double-blind 3-arm randomized controlled trial & 1 & Hamilton et al $^{9}$ \\
\hline
\end{tabular}


Table 2 Characteristics of Included Studies

\begin{tabular}{|c|c|c|c|}
\hline & Hamid et al $^{1}$ & Hamilton et al $^{9}$ & Reurink et al ${ }^{10,11}$ \\
\hline Study design & Single-blind randomized controlled trial & $\begin{array}{l}\text { Double-blind 3-arm randomized con- } \\
\text { trolled trial }\end{array}$ & $\begin{array}{l}\text { Double-blind randomized placebo- } \\
\text { controlled trial }\end{array}$ \\
\hline $\begin{array}{l}\text { Level of } \\
\text { evidence }\end{array}$ & II & II & II \\
\hline Validity $^{a}$ & 7 & 9 & 8 \\
\hline
\end{tabular}

Participants 28 participants (median age $21 \mathrm{y} ; 85.7 \%$ men, $53.6 \%$ national level athletes) with acute ( $<7 \mathrm{~d}$ since injury onset) grade $2 \mathrm{a}$ hamstring injury (mean 4.6 [2.15] d; $50 \%$ recurrent, $50 \%$ new)

Intervention Single intralesion 3-mL PRP injection investigated under ultrasound guidance + rehabilitation program (PATS)

Control PATS rehabilitation program

intervention

Outcome measure(s)

Results

Main findings/ conclusions
- Time to RTP: time (days) from data of injury onset until patient fulfilled criteria to RTP

- Subjective pain severity scores (BPI$\mathrm{SF})$

- Subjective pain interference scores (BPI-SF)
- PRP group had significantly lower pain severity scores than controls at all points

- Difference in pain interference scores between groups not statistically significant, though pain intensity lower at all time points in PRP group

A single 3-mL injection of autologous PRP combined with a PATS rehabilitation program was significantly more effective than a control in reducing the severity of pain and allowing a significantly shorter time to return to play after an acute grade 2 a hamstring injury
90 male professional athletes (18-50 y, mean 25.9 [5.8]) with MRI positive acute (within $5 \mathrm{~d}$ of onset of injury; recurrent/chronic excluded) grade I/II hamstring injuries

Three 1-mL PRP injections + rehabilitation $(5 \times / \mathrm{wk})$

PPP injection control + rehabilitation $(5 \times / \mathrm{wk})$

No injection parallel control + rehabilitation

Rehabilitation: ROM, progressive strengthening exercises, core stability training, agility exercises, sport-specific functional field testing

- Time to RTS: successful and asymptomatic completion of progressive criteria-based rehabilitation, clinical evaluation, and isokinetic

- Reinjury rate: acute hamstring strain at same site occurring within either 2 or 6 mo from RTS

- Isokinetic strength

- Alteration of MRI (T2 intensity)

- Median RTS 21 d (95\% CI, 17.9-24.1) PRP vs $27 \mathrm{~d}$ (95\% CI, 20.6-33.4) PPP vs 25 d (95\% CI, 21.5-28.5) control

- At any time, PRP patient has $2.29 \times$ greater chance of RTP compared with patient given PPP

- No significant difference in reinjury rate, isokinetic strength, or MRI alterations

80 competitive and recreational athletes (18-50 y) with acute grade I/II hamstring injuries as confirmed by MRI

Two 3-mL intramuscular PRP injections (first $5 \mathrm{~d}$ after injury, second 5-7 d later) + rehabilitation program (not specified)

Saline placebo injection + rehabilitation program (not specified)

- Time to return to play: time until patients could resume sports activity during 6 mo of follow-up

- Reinjury rate at 1-y follow-up

- Alteration in clinical and MRI parameters

- Subjective patient satisfaction and perceived recovery, NPRS

- Flexibility: active knee extension, passive SLR

- Isometric knee-flexion dynamometry

- Hamstring outcome score

- Median time to RTS 42 d (IQR 30-58) PRP vs 42 d (IQR 37-56) placebo (95\% CI, 0.61-1.51; $P=.66$ )

- $27 \%$ in PRP group sustained reinjury during first year follow-up period vs $30 \%$ in placebo group

- No significant differences between groups on subjective pain satisfaction, perceived recovery, numeric pain scale, and overall hamstring outcome score, nor in measured flexibility or strength deficits

There is no benefit of a single PRP injection over an intensive rehabilitation program in professional athletes who have sustained acute, MRI positive hamstring injuries
Intramuscular PRP injections showed no benefit compared with placebo injections in patients with acute hamstring injuries in the time to return to play nor in the subjective, clinical, and MRI measures

Abbreviations: BPI-SF, Brief Pain Index-Short Form; CI, confidence interval; IQR, interquartile range; MRI, magnetic resonance imaging; NPRS, Numeric Pain Rating Scale; PATS, progressive agility and trunk stability; PRP, platelet-rich plasma; PPP, platelet-poor plasma; ROM, range of motion; RTP, return to play; RTS, return to sport; SLR, straight leg raise.

${ }^{\mathrm{a}}$ PEDRo (Physiotherapy Evidence Database) scale maximum score: 10. 
$(\mathrm{N}=28)$, however, demonstrated that PRP injections combined with a rehabilitation program allowed significantly higher levels of pain relief, faster regeneration, and shorter recovery time following an acute hamstring injury than rehabilitation alone. ${ }^{1}$ It is important to note that in this study, the control group demonstrated a very large SD for time to return to play (42.5 [20.6] d vs 26.7 [7.0] d for the PRP group). This was not discussed in the article, but the high SD would signify a large variation in the times to return to play in the control group and suggests that at least 1 subject in the control group had a significantly longer recovery time as compared to the rest of the subjects, which may have negatively skewed the data for the control group.

Based on the conflicting and limited existing evidence, it cannot be determined that PRP injections have value in treating acute hamstring injuries as compared to rehabilitative therapy alone. However, some low-level evidence case studies and case series of varying sizes and participant demographics, as well as a randomized comparison study involving general muscle injury, and a retrospective review involving proximal hamstring injury, have all shown positive effects of PRP injections in hamstring injuries as compared with rehabilitation alone. $3,4,8,18$

Furthermore, the articles included in this CAT suggest that age and severity of injury are 2 factors that may be affecting PRP effects on hamstring injury outcomes. The study supporting PRP injection ${ }^{1}$ included subjects with a median age of 21 years, as compared to the other 2 studies whose subjects ranged from 18 to 50 years old, with only 1 study $^{9}$ reporting a mean age (25.9 [5.8] y). Furthermore, PRP injection ${ }^{1}$ may be supported in acute grade IIa hamstring injuries, or those with recurrent injuries as opposed to lower severity injuries. These differences may suggest that if PRP injections are to be tried, it may be more beneficial for younger athletes-potentially high school and college-aged-who compete at a higher level of competition and who have sustained more severe acute hamstring injuries, or recurrent hamstring injuries. Future research should aim to establish clear, consistent conclusions in regard to the effectiveness of PRP in specific patient demographics and injury severities, as well as investigate the optimal parameters of PRP injections and long-term effects.

It is important to note that in addition to the limitations in depth of existing literature, the 3 studies included in this CAT have several limitations, including variations between author definitions of rehabilitation programs and recovery time. The rehabilitation or conservative treatments used as comparison groups, outlined in Table 2, differed among the 3 studies. Similarly, the authors' definitions of recovery time differed slightly among the articles. One article ${ }^{10,11}$ generally defined recovery time as the time (measured in days) until patients resumed sport activities, while 2 articles ${ }^{1,9}$ defined recovery time as the number of days from injury until the patient met criteria to be cleared to return to play. Criteria outlined by Hamilton et $\mathrm{al}^{9}$ included successful and asymptomatic completion of progressive criteria based on rehabilitation progression, clinical evaluation, and interpretation of isokinetic testing, while criteria outlined by Hamid et al $^{1}$ included pain-free hamstring palpation and contraction, symmetrical active knee extension range of motion, and hamstring strength within $10 \%$ upon bilateral comparison.

Furthermore, the articles in this CAT have limitations both in their generalizability and methods of PRP injection administration. Two articles included males, either predominantly or exclusively, ${ }^{1,9}$ while 1 did not specify participant gender. ${ }^{10,11}$ It is unclear at current time if gender differences exist in regard to effects of PRP administration or in recovery time following hamstring injury, which served as the primary outcome measure.
However, hamstring injury has been shown to occur more frequently in males than females, with males also experiencing a higher incidence of recurrent injury. ${ }^{6}$ Only 1 article ${ }^{9}$ discussed participant sport involvement (football); however, participant positions within the sport were not provided. Since sport participation was not discussed in these articles, or in other existing studies, it cannot be determined if sport discipline and/or position will affect a patient's outcome or response to PRP. However, hamstring injuries have been shown to be more common in men's football, as well as in men's and women's soccer. ${ }^{6}$ Only 1 article provided mean or median ages in the early $20 \mathrm{~s},{ }^{1}$ while 1 had a mean age in the mid- to late-20s, ${ }^{9}$ and 1 only specified that inclusion criteria required participants be at least 18 years old. ${ }^{10,11}$ This is important to note since literature has suggested that age can affect muscle healing. ${ }^{19}$ Also, the article that supported PRP injection was the only 1 to use subjects with a mean age in the early 20 s. Finally, all of the articles included only acute grade I or II hamstring injuries. However, 2 of the articles grouped grade I and II strains into the same cohort, ${ }^{9-11}$ while 1 involved grade II only, ${ }^{1}$ so the effect of PRP injection on various grades of hamstring injury is not clear. However, the study that included grade II hamstring strains exclusively was the only article in this CAT to show benefits of PRP. It is also important to note that research has demonstrated that not all hamstring injuries are the same-different types of hamstring injuries vary based on the muscle involved, type of muscle damage, and mechanism of injury - and can differ in the required duration of rehabilitation and recovery time. ${ }^{20}$

Similarly, the different studies included in this CAT were not consistent in the parameters utilized for PRP injection procedure, including number of injections, amount of PRP per injection, location of injection, and amount of time between injury and injection. One study ${ }^{1}$ administered single injections to participants, while the other administered multiple injections. ${ }^{9-11}$ Two studies administered 3-mL of PRP per injection, however, 2 administered the injections directly into the lesion, ${ }^{1,9}$ while the other provided intramuscular administration. ${ }^{10,11}$ Only 2 of the articles specified the time between injury and injection: 1 study injected within a week of injury (mean of 4.6 [1.94] d) ${ }^{1}$ while the other injected within 5 days of injury (median $3 \mathrm{~d}$ ). ${ }^{10,11}$ Controversy exists over the optimal window between injury and administration of PRP injection. ${ }^{21}$ Finally, none of the articles specified the concentration of platelets and growth factors within the PRP or the time between injection and the start of rehabilitation.

Based on these external validity limitations, future studies should be conducted to establish a clear, consistent consensus regarding the appropriate patient demographics and parameters for optimal PRP efficacy. Since age can affect the healing process, future studies should compare various patient age groups to determine if age affects the value of PRP injections. ${ }^{19}$ Future studies should also separate grade I and II injuries into different cohorts to more intensively compare the effects of PRP on different grade injuries. Furthermore, because muscle can heal differently in recurrent injuries due to structural changes after initial acute injuries, future research should focus on PRP effects in recurrent hamstring injury. ${ }^{22}$ In addition, future research should aim to develop a universal, standardized protocol of optimal parameters for PRP injections, including number of injections, amount of PRP per injection, concentration of platelets and growth factors in a PRP injection, location of injection, time between injury onset and injection, and time between injection and the beginning of rehabilitation. Finally, long-term research should be conducted beyond a 1-year follow-up to investigate the long-term effects of PRP on 
muscle health, functionality, patient satisfaction, as well as to identify any long-term adverse effects of PRP injection.

While this study focused on recovery time of a hamstring injury as the primary outcome, another significant concern for athletes suffering a hamstring injury is the high-recurrence rate. Existing literature is limited and does not appear to show benefits of PRP injections in regard to reducing reinjury rate. However, current studies suggest that incorporating eccentric strengthening into the rehabilitation program may help to reduce the risk of recurrence following hamstring injury. ${ }^{23}$ Future long-term research should be conducted to further investigate the effects of PRP injections, as well as the combination of PRP injections and an eccentric strengthening program in regard to reducing reinjury rate following a hamstring injury.

In conclusion, future research needs to be conducted to further investigate PRP injections as an additional treatment option for hamstring injury. At present time, while no adverse effects have been reported, PRP injections cannot be recommended as having value for hamstring injuries as compared to rehabilitative therapy alone, due to limited and conflicting evidence in current literature.

\section{References}

1. Hamid MSA, Mohamed Ali MR, Yusof A, George J, Lee LP. Platelet-rich plasma injections for the treatment of hamstring injuries: a randomized controlled trial. Am J Sport Med. 2014;42(10):24102418. PubMed ID: 25073598 doi:10.1177/0363546514541540

2. Orchard J, Seward H. Epidemiology of injuries in the Australian Football League, seasons 1997-2000. Br J Sports Med. 2002;36:39_ 44. PubMed ID: 11867491 doi:10.1136/bjsm.36.1.39

3. Rettig AC, Meyer S, Bhadra AK. Platelet-rich plasma in addition to rehabilitation for acute hamstring injuries in NFL players: clinical effects and time to return to play. Orthop J Sports Med. 2013;1(1):2325967113494354. PubMed ID: 26535233 doi:10. 1177/2325967113494354

4. Wetzel RJ, Ronak MP, Terry MA. Platelet-rich plasma as an effective treatment for proximal hamstring injuries. Orthopedics. 2013; 36(1):e64-e70. PubMed ID: 23276355 doi:10.3928/01477447-2012 1217-20

5. Sherry MA, Johnston TS, Heiderscheit BC. Rehabilitation of acute hamstring strain injuries. Clin Sports Med. 2015;34(2):263-284. PubMed ID: 25818713 doi:10.1016/j.csm.2014.12.009

6. Dalton SL, Kerr ZY, Dompier TP. Epidemiology of hamstring strains in 25 NCAA sports in the 2009-2010 to 2013-2014 academic years. Am J Sport Med. 2015;43(11):2671-2679. PubMed ID: 26330571 doi:10.1177/0363546515599631

7. Moraes VY, Lenza M, Tamaoki MJ, Faloppa F, Belloti JC. Platelet-rich therapies for musculoskeletal soft tissue injuries. Cochrane Database Syst Rev. 2014;(4):CD010071. PubMed ID: 24782334 doi:10.1002/14651858.CD010071.pub3

8. Bubnov R, Yevseenko V, Semeniv I. Ultrasound guided injections of platelets rich plasma for muscle injury in professional athletes. Comparative study. Med Ultrason. 2013;15(2):101-105. PubMed ID: 23702498 doi:10.11152/mu.2013.2066.152.rb1vy2
9. Hamilton B, Tol JL, Almusa E, et al. Platelet-rich plasma does not enhance return to play in hamstring injuries: a randomised controlled trial. Br J Sports Med. 2015;49:943-950. PubMed ID: 26136179 doi:10.1136/bjsports-2015-094603

10. Reurink G, Goudswaard GJ, Moen MH, et al. Rationale, secondary outcome scores and 1-year follow up of a randomised trial of platelet-rich plasma injections in acute hamstring injury: the Dutch Hamstring Injection Therapy study. Br J Sports Med. 2015;49:1206-1212. PubMed ID: 25940636 doi:10.1136/bjsports2014-094250

11. Reurink G, Goudswaard GJ, Moen MH, et al. Platelet-rich plasma injections in acute muscle injury. $N$ Engl $\mathrm{J}$ Med. 2014;370(26):2546-2547. PubMed ID: 24963588 doi:10.1056/ NEJMc1402340

12. World Anti-Doping Agency. Prohibited list. WADA website. 2017. https://www.wada-ama.org/en/questions-answers/prohibited-list\# item-380. Accessed January 28, 2017.

13. Zanon G, Combi F, Combi A, Perticarini L, Sammarchi L, Benazzo F. Platelet-rich plasma in the treatment of acute hamstring injuries in professional football players. Joints. 2016;4(1):17-23. PubMed ID: 27386443 doi:10.11138/jts/2016.4.1.017

14. Martinez SF. Practical guidelines for using PRP in the orthopaedic office. AAOS Now. Oct 2015;9(10).

15. Shute N. Platelet-rich plasma therapy gains fans, but remains unproven. NPR. June 11, 2014. http://www.npr.org/sections/healthshots/2014/06/11/320971966/platelet-rich-plasma-therapy-gains-fansbut-remains-unproven. Accessed October 28, 2015.

16. Ebell MH, Siwek J, Weiss BD, et al. Strength of Recommendation Taxonomy (SORT): a patient-centered approach to grading evidence in the medical literature. Am Fam Physician. 2004;69(3):548-556. PubMed ID: 14971837

17. OCEBM Levels of Evidence Working Group. The Oxford 2011 levels of evidence. 2011. Oxford Centre for Evidence-Based Medicine. http://www.cebm.net/index.aspx?o=5653

18. Borrione P, Pereira Ruiz MT, Giannini S, Di Gianfrancesco A, Pigozzi F. Effect of platelet-released growth factors on muscle strains: a case control report. Medicina Dello Sport. 2011;64(3): 317-322.

19. Mall NA, Tanaka MJ, Choi LS, Paletta GA Jr. Factors affecting rotator cuff healing. J Bone Joint Surg Am. 2014;96(9):778-788. PubMed ID: 24806015 doi:10.2106/JBJS.M.00583

20. Brukner P. Hamstring injuries: prevention and treatment - an update. Br J Sports Med. 2015;49:1241-1244. PubMed ID: 26105015 doi:10. 1136/bjsports-2014-094427

21. Anitua E, Sanchez M, Padilla S. More on platelet-rich plasma injections in acute muscle injury. $N$ Engl J Med. 2014;371(13): 1264-1265. doi:10.1056/NEJMc1409204

22. Croisier JL. Factors associated with recurrent hamstring injuries. Sports Med. 2004;34(10):681-695. PubMed ID: 15335244 doi:10. 2165/00007256-200434100-00005

23. Tyler TF, Schmitt BM, Nicholas SJ, McHugh MP. Rehabilitation after hamstring strain injury emphasizing eccentric strengthening at long muscle lengths: results of long term follow up. J Sport Rehabil. 2017;26(2):131-140. PubMed ID: 27632842 doi:10.1123/jsr.20150099 